\title{
Older adults' neural activation in the reward circuit is sensitive to face trustworthiness
}

\author{
Leslie A. Zebrowitz ${ }^{1}$. Noreen Ward ${ }^{2}$ - Jasmine Boshyan ${ }^{1,2,3}$ • Angela Gutchess ${ }^{1}$. \\ Nouchine Hadjikhani ${ }^{2,4}$
}

Published online: 6 December 2017

(C) Psychonomic Society, Inc. 2017

\begin{abstract}
We examined older adult (OA) and younger adult (YA) neural sensitivity to face trustworthiness in reward circuit regions, previously found to respond to trustworthiness in YA. Interactions of face trustworthiness with age revealed effects exclusive to OA in the amygdala and caudate, and an effect that was not moderated by age in the dorsal anterior cingulate cortex (dACC). OA, but not YA, showed a nonlinear amygdala response to face trustworthiness, with significantly stronger activation response to high than to medium trustworthy faces, and no difference between low and medium or high. This may explain why an earlier study investigating OA amygdala activation to trustworthiness failed to find a significant effect, since only the linear low versus high trustworthiness difference was assessed. OA, but not YA, also showed significantly stronger activation to high than to low trustworthy faces in the right caudate, indicating a positive linear effect, consistent with previous YA research, as well as significantly stronger activation to high than to medium but not low trustworthy faces in the left caudate, indicating a nonlinear effect. Activation in dACC across both age groups showed a positive linear effect consistent with previous YA research. Finally, OA rated the faces as more
\end{abstract}

Leslie A. Zebrowitz

zebrowitz@brandeis.edu

1 Department of Psychology, MS 062, Brandeis University, Waltham, MA 02453, USA

2 Athinoula A. Martinos Center for Biomedical Imaging, Massachusetts General Hospital, Charlestown, MA 02129, USA

3 Department of Radiology, Harvard Medical School, Boston, MA 02129, USA

4 Gillberg Neuropsychiatry Center, University of Gothenburg, Gothenburg, Sweden trustworthy than did YA across all levels of trustworthiness. Future research should examine whether the null effects for YA were due to our inclusion of older faces. Research also should investigate possible implications of our findings for more ecologically valid OA responses to people who vary in facial trustworthiness.

Keywords Aging $\cdot$ Reward $\cdot$ Amygdala $\cdot$ Face trustworthiness

There is a widespread belief that older adults (OA) are more vulnerable to consumer fraud than are younger adults (YA) (Carrns, 2017). Indeed, this vulnerability has been codified in laws, such as a Wisconsin provision of additional punishment to those who take advantage of the elderly for violations of consumer laws involving false advertising, motor vehicle repair, home improvement, and telecommunication services, among others (Violations Against Elderly or Disabled Persons, 2012 ). However, it has been observed that much of the evidence supporting the belief in greater vulnerability in $\mathrm{OA}$ is anecdotal, and that representative surveys show that $\mathrm{OA}$ are actually less likely to report experiencing consumer fraud than are adults of other ages (Ross, Grossman, \& Schryer, 2014). Of course, self-report may be biased (Oliveira et al., 2017), and there is some experimental evidence to suggest greater vulnerability in OA. OA women, but not men, were more likely than YA to click on phishing e-mails (Oliveira et al., 2017), and there is other evidence that OA were less accurate than YA in detecting deception, with OA poorer recognition of negative emotional expressions mediating this effect (Ruffman, Murray, Halberstadt, \& Vater, 2012; Stanley \& Blanchard-Fields, 2008). Additional research consistent with the idea that OA would be more vulnerable to fraud has shown that, compared with YA, OA gave more positive ratings to 
faces on dangerousness/trustworthiness continua, an effect that was stronger for the most dangerous/least trustworthy faces in some cases (Castle et al., 2012; Ruffman, Sullivan, \& Edge, 2006; Zebrowitz, Franklin, Hillman, \& Boc, 2013), but held true across all faces in others (Zebrowitz, Boshyan, Ward, Gutchess, \& Hadjikhani, 2017). In addition, Castle et al. (2012) reported that OA not only rated low trustworthy faces more positively than YA but also failed to show amygdala and insula activation to those faces. The present study sought to replicate and extend the Castle et al. (2012) study. Specifically, we investigated OA and YA neural sensitivity to face trustworthiness in additional brain regions that responded to face valence in a meta-analysis of YA responses across variations in face trustworthiness and attractiveness (Mende-Siedlecki, Said, \& Todorov, 2013).

Castle et al. (2012) examined age differences in the neural response to variations in trustworthiness in the amygdala and anterior insula. They found greater YA bilateral anterior insula activity for faces judged as untrustworthy than those judged as trustworthy, but no effect for OA, and no effects for either YA or OA in the amygdala. The focus on these two regions is consistent with meta-analytic evidence that each responds to face trustworthiness in YA (Mende-Siedlecki et al., 2013; Santos, Albeida, Oliveiros, \& Castelo-Branco, 2016). However, the meta-analyses found not only a negative linear effect of face trustworthiness on amygdala activation but also a nonlinear effect, with stronger activation to low or high than to medium trustworthy faces. Thus, it is possible that the Castle et al. (2012) study failed to find effects for amygdala activation because they only compared low to high trustworthy faces rather than investigating the nonlinear effect. Consistent with this suggestion, a study examining amygdala response to emotional pictures found that OA, like YA, showed stronger activation to positive and negative images than to neutral ones (Mather et al., 2004). In addition to effects on amygdala activation, the Mende-Siedlecki et al. (2013) meta-analysis revealed a negative linear effect of face trustworthiness/attractiveness on right insula activation but a positive linear effect on left insula activation. Surprisingly, Castle et al. (2012) reported the strongest negative linear effect for YA in left insula and no effect for OA in either hemisphere.

The present study extended the Castle et al. (2012) investigation by including medium in addition to high and low trustworthy faces in order to detect nonlinear effects, and by including hemisphere as a factor in our analyses. It should be noted that the inclusion of medium trustworthy faces not only can uncover nonlinear patterns of activation, but also the information that medium faces can provide bears on the question of whether neural activation to face trustworthiness parallels research documenting an OA positivity effect. This positivity effect is shown in less processing of negative stimuli and/or greater processing of positive stimuli as compared with YA (Reed \& Carstensen, 2012), and it has been documented in research on attention and memory (Isaacowitz \& Blanchard-Fields, 2012; Murphy \& Isaacowitz, 2008; Reed, Chan, \& Mikels, 2014) as well as evaluations of evocative pictures (D. P. Smith, Hillman, \& Duley, 2005), emotionally laden words (Kensinger, 2008), faces varying in emotion expression (Czerwon, Lüttke, \& Werheid, 2011; Riediger, Voelkle, Ebner, \& Lindenberger, 2011), and neutral expression faces (Castle et al., 2012; Zebrowitz et al., 2017; Zebrowitz et al., 2013). In addition to this behavioral evidence for OA positivity, neural evidence has been provided in amygdala responses to pictures varying in emotional valence. Specifically, Mather et al. (2004) found not only the abovenoted nonlinear amygdala response to these pictures in YA and OA but also stronger OA activation to positive than to negative images and stronger YA activation to negative than to positive ones.

In addition to including medium trustworthy faces, we examined five other brain regions that showed YA responsiveness to face valence in the Mende-Siedlecki et al. (2013) metaanalysis of 29 studies that included 12 manipulating trustworthiness and 17 manipulating attractiveness. These additional regions were the dorsal anterior cingulate cortex (dACC), caudate, medial orbital frontal cortex (mOFC), nucleus accumbens (NAcc), and ventral medial prefrontal cortex (vmPFC), all of which showed positive linear effects of face trustworthiness/attractiveness in YA. Some reason to expect similar effects for $\mathrm{OA}$ is provided by the finding that, for OA as well as YA, smiling faces presented as a reward for correct task performance, elicited more activation in caudate and NAcc than did frowning faces, presented as a punishment (Drueke et al., 2015). However, it may be that the neural response to the smiling and frowning faces derived more from the positive or negative feedback they provided regarding the participants' performance than from the valence of the faces per se. In the present study, face trustworthiness was not associated with an extrinsic reward and only had intrinsic reward value.

It should be noted that there is reason to expect nonlinear effects of face trustworthiness not only in the amygdala but also in the additional regions identified in the MendeSiedlecki et al. (2013) meta-analysis. These regions are part of the neural reward network (Liu, Hairston, Schrier, \& Fan, 2011; Sescousse, Caldu, Segura, \& Dreher, 2013), whose function is to guide goal-related behavioral responses, such as approach and avoidance (Delgado, 2007). Consistent with this function in the case of face trustworthiness are the results of a mouse-tracking methodology that displayed approachability decisions via the movement trajectories of a computer mouse (Hehman, Stolier, \& Freeman, 2015) and revealed greater approach toward trustworthy than toward untrustworthy faces (Martens, Hasinski, Andridge, \& Cunningham, 2012). In addition, animal research using single cell recordings suggests that these regions aid goal-directed behavior by 
processing both the appetitive and aversive qualities of stimuli, as evidenced by reward circuit responses to both positively and negatively valenced stimuli (Hayes, Duncan, $\mathrm{Xu}, \&$ Northoff, 2014; Hosokawa, Kato, Inoue, \& Mikami, 2007; Namburi, Al-Hasani, Calhoon, Bruchas, \& Tye, 2016; Nishijo, Ono, \& Nishino, 1988). Individual human studies also have demonstrated nonlinear responses in reward circuit regions of interest (ROIs), including ACC, caudate, mOFC, and NAcc (Elliott, Newman, Longe, \& Deakin, 2003; Liang, Zebrowitz, \& Zhang, 2010; Winston, O’Doherty, Kilner, Perrett, \& Dolan, 2007). Mende-Siedlecki et al. (2013) reported that their meta-analytic assessment, which found nonlinear responses only in amygdala, was underpowered because research on human neural responses to faces varying in attractiveness or trustworthiness has most often examined linear effects. Our inclusion of medium trustworthy faces enabled us to look for nonlinear patterns of activation.

In addition to including a medium trustworthy face category and examining age differences in neural activation to face trustworthiness in the broader reward circuit, we modified the Castle et al. (2012) paradigm by including equal numbers of older and younger faces within each of three female face trustworthiness categories. Castle et al. (2012) did not control the distribution of face age and sex across variations in face trustworthiness, which makes it possible that their findings may have reflected age differences in responses to face age and/or sex rather than in responses to low trustworthiness per se. For example, an own-age advantage has been documented in behavioral studies of face recognition (Anastasi \& Rhodes, 2005; Fulton \& Bartlett, 1991; Perfect \& Harris, 2003; Wright \& Stroud, 2002), age recognition (Voelkle, Ebner, Lindenberger, \& Riediger, 2012), and emotion recognition (Folster, Hess, Huhnel, \& Werheid, 2015; Malatesta, Izard, Culver, \& Nicolich, 1987; Riediger et al., 2011). Moreover, an own-age advantage also has been shown in greater neural activation to own-age than other-age facial expressions in several reward regions (Ebner et al., 2013). Notably, however, an own-age disadvantage has been found for OA in the case of deception detection. OA, but not YA, were more likely to believe that an own-age liar was telling the truth rather than an other-age liar (Slessor, Phillips, Ruffman, Bailey, \& Insch, 2014).

If the OA positivity effect is shown in neural activation to variations in face trustworthiness in the reward region, then one might expect stronger effects of high versus medium or low trustworthiness for OA than YA and stronger effects of low versus medium or high trustworthiness for YA than OA. Although one might also expect OA to show lower activation than YA in regions that are preferentially activated by low face trustworthiness, and higher activation than YA in regions that are preferentially activated by high face trustworthiness, caution is required in interpreting main effects of age, which may reflect age-related changes in the hemodynamic responses that are not specific to effects of face trustworthiness (Buckner, Snyder, Sanders, Raichle, \& Morris, 2000; D’Esposito, Zarahn, Aguirre, \& Rypma, 1999). We therefore tested the following predictions:

1. Neural activation would vary with face trustworthiness in the ROIs that showed such effects for YA in previous meta-analyses.

2. YA would show stronger activation to low than to medium or high trustworthy faces in regions preferentially activated by low trustworthy faces, the amygdala and right insula, while OA would not, in keeping with an OA positivity effect.

3. Both YA and OA would show a nonlinear response to face trustworthiness in the amygdala, with stronger activation to low and high trustworthy faces than to medium, consistent with the meta-analytic results for YA. However, the effect for high versus medium trustworthy faces would be strongest for OA and the effect for low versus medium would be strongest for YA, consistent with an OA positivity effect.

4. Both YA and OA would show stronger activation to high than to medium or low trustworthy faces in regions preferentially activated by high trustworthy faces, the dACC, caudate, left insula, mOFC, and vmPFC, and these effects may be stronger for OA, consistent with an OA positivity effect. Stronger activation to high than to medium and low trustworthiness would indicate a linear effect, while stronger activation to high than medium, but not low, trustworthiness would indicate a nonlinear effect.

5. The OA positivity effect also would be shown in higher trustworthy ratings by OA than YA.

\section{Method}

\section{Participants}

Participants were 24 YA ( 12 men) ages 19 to 32 years $(M=$ 24.08 years, $S D=3.01)$ and $24 \mathrm{OA}(13 \mathrm{men})$ ages 65 to 88 years $(M=72.83$ years, $S D=7.22)$. One YA woman did not complete the face ratings described below. Participants were paid $\$ 65$ for participating in the fMRI scanning as well as completing the face ratings and control measures. Unfortunately, sufficient data were not available to perform a proper power analysis to determine sample size. The only study that included both younger and older participants with $N$ $=21$ YA and $N=23$ OA (Castle et al., 2012) did not report any statistics from which a power analysis could be performed. Of the 24 studies examining the YA neural response to face trustworthiness across the two literature reviews with an average $N$ 
$=20.08$, only four provided the necessary information to perform a power analysis. The results for those four studies revealed that for $80 \%$ power at a .05 alpha level, the sample size per group was estimated to be 27 in a two-group repeatedmeasures high/low trustworthiness design.

\section{Sample descriptive measures}

OA were screened using the Mini-Mental State Examination (Folstein, Folstein, \& McHugh, 1975), all scoring above 26 out of $30(M=29.44, S D=.99)$. Measures of vision, affect, and cognitive function were also administered to all participants to ascertain the representativeness of our sample. Results showed that OA performed worse than YA on tests of visual acuity, contrast sensitivity, and processing speed (Pattern Comparison Test) and scored higher on tests of vocabulary and positive affect, consistent with previous studies of community-dwelling older versus younger adults (see Table 1).

\section{Face stimuli}

To select face stimuli varying in trustworthiness, we conducted a pretest on Amazon Mechanical Turk (MTurk: https:// requester.mturk.com/). Participants were paid $\$ 1$ per 15 minutes of participation to make trustworthy ratings of 272 female faces, using 7-point scales, with endpoints labeled not at all and very trustworthy. These photos were selected from three publicly available face databases (Center for Vital Longevity Face Database: http://agingmind.utdallas.edu/ download-stimuli/face-database/; FACES database: https:// people.clas.ufl.edu/natalieebner/links/; and Karolinska
Directed Emotional Faces: http://www.emotionlab.se/ resources $/ \mathrm{kdef}$ ). A random order of pretest older and younger female faces was rated by 43 judges $(20$ males, mean age $=34.35$ years, $S D=13.29 ; 23$ females, mean age $=32.74$ years, $S D=10.61)$. Based on $z$ scores of the pretest ratings, we selected photos of 180 women ( 90 older, $M=75$. 22 years, $S D=6.47 ; 90$ younger, $M=23.97$ years, $S D=4.33$ ) with each age group equally represented in the high trustworthiness $(M=4.78, S D=.22)$, medium trustworthiness $(M=3.98, S D=.10)$, and low trustworthiness $(M=3.12, S D=.29)$ categories, with each category significantly different from the rest (all $p \mathrm{~s}<.001$ ). Medium face trustworthiness were assumed to be "neutral" with regard to trustworthiness (neither high nor low). They served to examine nonlinear responses in the reward circuit, which would be revealed in greater activation to high and low than to medium face trustworthiness.

We included both older and younger faces so that any perceiver age effects would not be confounded with a tendency to respond more strongly to own-age stimuli. Due to the nature of an fMRI block design, we could not analyze effects of face age because, as described below, both older and younger faces were included in the same block of faces. We used only female faces because including both sexes as well as both ages would have made a properly counterbalanced protocol too long for an fMRI session.

\section{$f M R I$}

Design and procedure Faces were presented in blocks of the same trustworthiness level (high, medium, low), with six faces per block, three younger and three older, with age randomly

Table 1 Sample descriptive information

\begin{tabular}{|c|c|c|c|c|c|c|}
\hline \multirow[t]{2}{*}{ Measure } & \multicolumn{2}{|c|}{ Younger adults } & \multicolumn{2}{|c|}{ Older adults } & \multirow[b]{2}{*}{$F$ value } & \multirow[b]{2}{*}{$p$ value } \\
\hline & $M$ & $S D$ & $M$ & $S D$ & & \\
\hline Snellen Visual Acuity (denominator) & 14.69 & 5.45 & 27.50 & 10.00 & 17.11 & $<.001$ \\
\hline Mars Letter Contrast Sensitivity (Mars Perceptrix, Chappaqua, NY) & 1.79 & .06 & 1.63 & .18 & 14.18 & .001 \\
\hline Benton Facial Recognition Test (Benton et al., 1983) & 48.00 & 3.37 & 45.96 & 5.72 & 2.10 & .154 \\
\hline Pattern Comparison Test (Salthouse, 1993) & 41.59 & 8.40 & 30.17 & 5.80 & 28.38 & $<.001$ \\
\hline Shipley Vocabulary Test (Shipley, 1946) & 32.62 & 2.89 & 35.70 & 2.62 & 13.73 & .001 \\
\hline BCST Correct responses (Piper et al., 2012)* & 38.43 & 2.41 & 31.48 & 7.60 & 17.52 & $<.001$ \\
\hline BCST Perseverative errors* & 5.70 & 1.15 & 6.96 & 3.62 & 2.53 & .119 \\
\hline BCST Nonperseverative errors* & 3.87 & 2.05 & 9.70 & 8.26 & 10.76 & .002 \\
\hline BCST Trials to complete first category* & 9.48 & 2.13 & 12.17 & 8.26 & 2.30 & .137 \\
\hline PANAS Negative Affect (Watson et al., 1988) & 12.27 & 3.28 & 10.71 & 1.45 & 3.76 & .059 \\
\hline PANAS Positive Affect (Watson et al., 1988) & 26.91 & 6.65 & 32.62 & 7.53 & 6.96 & .012 \\
\hline
\end{tabular}

$* \mathrm{BCST}=$ Berg Card Sort Task, a validated version of the Wisconsin Card Sort Task. $N \mathrm{~s}=23$ in each age group except that Snellen data were missing for 10 younger and seven older participants; Mars data were missing for three younger and one older participants; Benton data were missing for one younger participant. Shipley data were missing for two younger participants. 
distributed within the block. Blocks were counterbalanced so that the same level of trustworthiness never occurred in succession, and each level of trustworthiness appeared once every three blocks. There were two runs, each including 15 blocks of faces (five high, five medium, five low trustworthiness) and eight blocks of fixation. Each face was presented for $1,800 \mathrm{~ms}$ following 200-ms fixation, for a total block duration of $12 \mathrm{~s}$ and a total run duration of 4.7 minutes.

Participants were told that they were going to see a number of faces and that after each face a red cross would appear. However, every so often, this cross would be blue instead of red. Participants were instructed to press the button every time they saw a blue cross. The purpose of the red cross was to focus participants on the eye region of the faces, which yields stronger neural responses (Hadjikhani et al., 2017). The blue cross was to maintain participants' attention to the task. In addition, they were asked to look at each face and think about how trustworthy it seemed to them.

Face stimuli presented during the fMRI scanning session were generated with a Dell Latitude E5440 computer, using EPrime 2.0, and back-projected via an LCD projector and a mirror attached to the head coil onto an acrylic rearprojection screen (DaTex, Da-Lite Corp.) providing a visually activated stimulus screen of $48^{\circ} \times 36^{\circ}$. Button-press responses were collected using an MR-compatible button box connected to the Dell computer via a custom USB interface. A trigger mechanism was used to lock stimulus presentation to the start of each TR during the fMRI scan.

Imaging data acquisition and analysis Anatomical and functional MR images were acquired with a 12-channel RF coil in a Siemens $3 \mathrm{~T}$ scanner (Siemens TrioTim, Erlangen) at the Martinos Center for Biomedical Imaging. The first scanning sequence consisted of Siemens's auto-align scout for the head allowing an automatic positioning and alignment of slices. Anatomical images were acquired using a multiecho magnetization prepared rapid gradient echo sequence (MEMPRAGE: matrix $=256 \times 256$; echo time $[\mathrm{TE}]:$ TE1 $=1.64$ $\mathrm{ms}$, TE2 $=3.5 \mathrm{~ms}$, TE3 $=5.36 \mathrm{~ms}$, TE4 $=7.22 \mathrm{~ms}$; repetition time $[\mathrm{TR}]=2,530 \mathrm{~ms}$; flip angle $=7^{\circ}$; slice thickness $=1.33$ $\mathrm{mm}$; in-plane resolution $=1 \mathrm{~mm} \times 1 \mathrm{~mm}$ ). Functional data were then acquired using an echo planar imaging (EPI) sequence (41 axial slices with 4-mm thickness; in plane resolution $=3.125 \mathrm{~mm} \times 3.125 \mathrm{~mm}$; matrix $=72 \times 72 ; \mathrm{FOV}=216$; $\mathrm{TE}=30 \mathrm{~ms} ; \mathrm{TR}=2,000 \mathrm{~ms}$; flip angle $=90^{\circ} ; 138$ time points) with a duration of 4.7 minutes.

Functional imaging data were preprocessed and analyzed with FSL (FEAT Version 5.98). Nonbrain tissue was removed from high-resolution anatomical images using FSL BET and fed into FEAT. Data were motion corrected using MCFLIRT and motion parameters added as confound variables to the model. Preprocessing further included spatial smoothing using a Gaussian kernel of $8 \mathrm{~mm}$. The final analysis comprised 24 subjects in the OA group and 24 in the YA group.

Activation to high, medium, and low face trustworthiness in each ROI was compared to baseline activation (to fixation crosses) for each participant using FILM with local autocorrelation correction. Registration to high-resolution structural images was carried out using FLIRT. Registration to Montreal Neurological Institute (MNI) standard space was then further refined using FNIRT nonlinear registration. Group-level analyses were carried out using mixedeffects GLM analysis using FLAME 1+2 with automatic outlier detection. In modeling subject variability, this kind of analysis allows inference about the population from which the subjects are drawn.

ROIs We examined seven a priori anatomical ROIs: amygdala, dACC, caudate, anterior insula, mOFC, NAcc, and vmPFC. ROIs were defined anatomically using the FSL Harvard-Oxford cortical and subcortical atlas. The dACC ROI was modified to include only the rostral portion, which is more implicated in reward processes than the caudal portion (Desikan et al., 2006). Each ROI was transformed from the MNI space into subject space, using nonlinear transformation with FSL FNIRT. For each subject, the value of the maximum contrast of parameter estimate (COPE) was extracted for the seven structures and each contrast of interest (low, medium, and high face trustworthiness), using the FSL featquery tool in FSL (S. Smith, 2002).

\section{Trustworthy ratings}

After scanning was completed, participants were shown the same faces out of the scanner but in a random order. They were asked to rate each face on a 7-point scale, with endpoints labeled not at all and very trustworthy. The protocol was 2 seconds of fixation, 2 seconds viewing the face, and 2 seconds to rate the face. All faces were rated twice, once with distraction and once with no distraction, with the order of distraction counterbalanced across participants. The distraction manipulation was included for another study and the data reported in this manuscript are for nondistraction ratings only.

\section{Results}

\section{Blood oxygen-level dependent (BOLD) response in ROIs}

\section{Overview}

We performed 3 (face trustworthiness: low, medium, high) $\times 2$ (participant age: YA, OA) $\times 2$ (hemisphere: left, right) mixeddesign ANOVAs on BOLD peak activation in the a priori 
ROIs. Face trustworthiness and hemisphere were withinsubjects factors. Although we have argued that either linear or nonlinear responses to face trustworthiness may be shown in the reward circuit, we report these separate effects only for the amygdala, where they were firmly established in the metaanalyses. We report the 2- $d f$ omnibus $F$ tests for the remaining ROIs. Although we report statistics and means for participant age main effects, we do not discuss these effects because, as noted in the introduction, they may reflect age-related changes in the hemodynamic response that are not specific to effects of face trustworthiness. Table 2 reports statistics for the ANOVA main effects and interactions. When significant at $p=.05$, we report comparisons between trustworthy conditions in the text to elucidate the pattern of the effect. Table 3 reports the means and standard errors for BOLD responses within each ROI as a function of face trustworthiness, participant age, and hemisphere.

\section{Amygdala}

There was no significant linear or quadratic effect of face trustworthiness or participant age on amygdala activation.
However, the predicted Face Trustworthiness $\times$ Participant Age interaction was significant. Comparisons between face trustworthiness levels within each age revealed that OA showed stronger amygdala activation to high than medium face trustworthiness, $p=.021$, but no significant differences between low and medium, $p=.109$, or low and high, $p=.393$, consistent with Hypotheses 2 and 3. However, contrary to these hypotheses, YA showed no significant difference between high and medium, $p=.333$, low and medium, $p=$ .444 , or low and high, $p=.090$, although the latter showed a trend toward a greater response to low trustworthiness, consistent with Hypothesis 2 (see Fig. 1a). Simple effects analyses within each age group supported these effects. Neither the linear nor the quadratic effect of face trustworthiness was significant for YA, linear $F(1,23)=2.16$, and quadratic $F(1,23)$ $=.03$, respective $p \mathrm{~s}=.155$ and $.874, \eta^{2}=.086$ and .001 . However, the quadratic effect was significant for OA, $F(1$, 23) $=4.63, p=.042, \eta^{2}=.168$, and the negative linear effect was not, $F(1,23)=1.22, p=.281, \eta^{2}=.050$. There also was a main effect for hemisphere, reflecting stronger activation on the right than on the left, but none of the interactions with hemisphere were significant.

Table 2 Face Trustworthiness $\times$ Participant Age $\times$ Brain Hemisphere ANOVAs for the examined ROIs

\begin{tabular}{|c|c|c|c|c|c|c|c|}
\hline & & Trustworthiness $(\mathrm{T})$ & Age (A) & Hemisphere $(\mathrm{H})$ & $\mathrm{A} \times \mathrm{T}$ & $\mathrm{H} \times \mathrm{T}$ & $\mathrm{H} \times \mathrm{A} \times \mathrm{T}$ \\
\hline \multirow[t]{3}{*}{ Amygdala (linear) } & $F(\mathrm{df})$ & $.38(1,46)$ & $.01(1,46)$ & $4.77(1,46)$ & $3.24(2,92)$ & $1.99(2,92)$ & $.04(2,92)$ \\
\hline & $\mathrm{p}$ & .541 & .935 & .034 & .044 & .142 & .960 \\
\hline & partial $\eta 2$ & .008 & $<.001$ & .094 & .066 & .042 & .001 \\
\hline \multirow[t]{3}{*}{ Amygdala (quadratic) } & $F(\mathrm{df})$ & $2.4(1,46)$ & & & & & \\
\hline & $p$ & .125 & & & & & \\
\hline & partial $\eta 2$ & .050 & & & & & \\
\hline \multirow[t]{3}{*}{ dACC } & $F(\mathrm{df})$ & $3.26(2,92)$ & $3.77(1,46)$ & & $.59(2,92)$ & & \\
\hline & $p$ & .043 & .058 & & .555 & & \\
\hline & partial $\eta 2$ & .066 & .076 & & .013 & & \\
\hline \multirow[t]{3}{*}{ Caudate } & $F(\mathrm{df})$ & $2.12(2,92)$ & $.39(1,46)$ & $.09(1,46)$ & $.42(2,92)$ & $2.44(2,92)$ & $3.41(2,92)$ \\
\hline & $p$ & .126 & .536 & .768 & .657 & .093 & .037 \\
\hline & partial $\eta 2$ & .044 & .008 & .002 & .009 & .050 & .069 \\
\hline \multirow[t]{3}{*}{ Insula } & $F(\mathrm{df})$ & $1.62(2,92)$ & $.63(1,46)$ & $.02(1,46)$ & $.33(2,92)$ & $.69(2,92)$ & $1.48(2,92)$ \\
\hline & $p$ & .204 & .432 & .878 & .721 & .506 & .233 \\
\hline & partial $\eta 2$ & .034 & .013 & .001 & .007 & .015 & .031 \\
\hline \multirow[t]{3}{*}{$\mathrm{mOFC}$} & $F(\mathrm{df})$ & $2.71(2,92)$ & $3.64(1,46)$ & $15.65(1,46)$ & $1.84(2,92)$ & $1.98(2,92)$ & $.23(2,92)$ \\
\hline & $p$ & .072 & .063 & $<.001$ & .165 & .144 & .793 \\
\hline & partial $\eta 2$ & .056 & .073 & .254 & .038 & .041 & .005 \\
\hline \multirow[t]{3}{*}{ Nacc } & $F(\mathrm{df})$ & $2.95(2,92)$ & $1.07(1,46)$ & $.87(1,46)$ & $.23(2,92)$ & $.54(2,92)$ & $.25(2,92)$ \\
\hline & $p$ & .057 & .306 & .355 & .798 & .588 & .778 \\
\hline & partial $\eta 2$ & .060 & .023 & .019 & .005 & .011 & .005 \\
\hline \multirow[t]{3}{*}{ vmPFC } & $F(\mathrm{df})$ & $2.23(2,92)$ & $.46(1,46)$ & $14.04(1,46)$ & $.40(2,92)$ & $1.97(2,92)$ & $1.45(2,92)$ \\
\hline & $p$ & .113 & .500 & $<.001$ & .670 & .145 & .239 \\
\hline & partial $\eta 2$ & .046 & .010 & .234 & .009 & .041 & .031 \\
\hline
\end{tabular}

Note. All ROIs are bilateral 


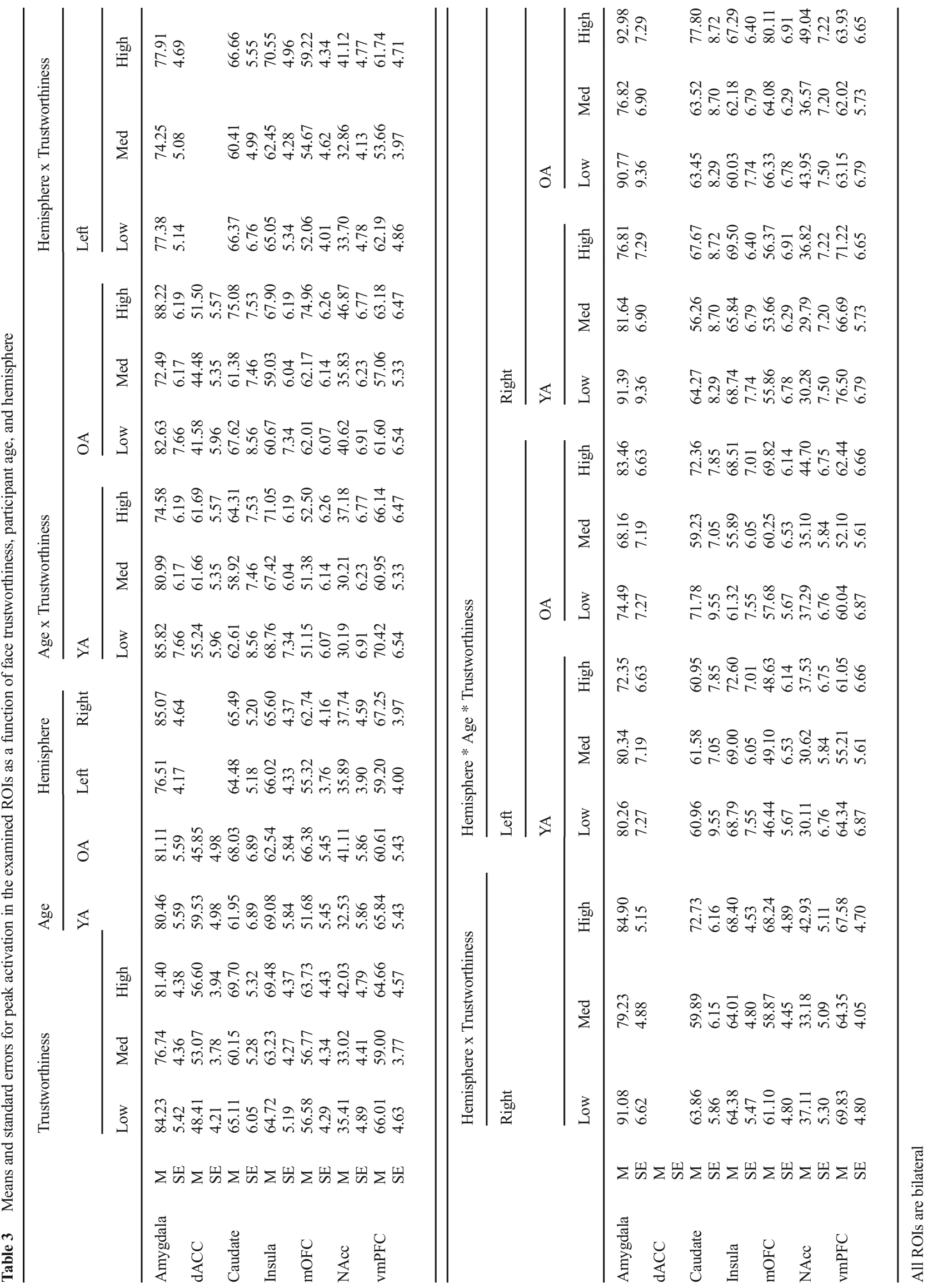




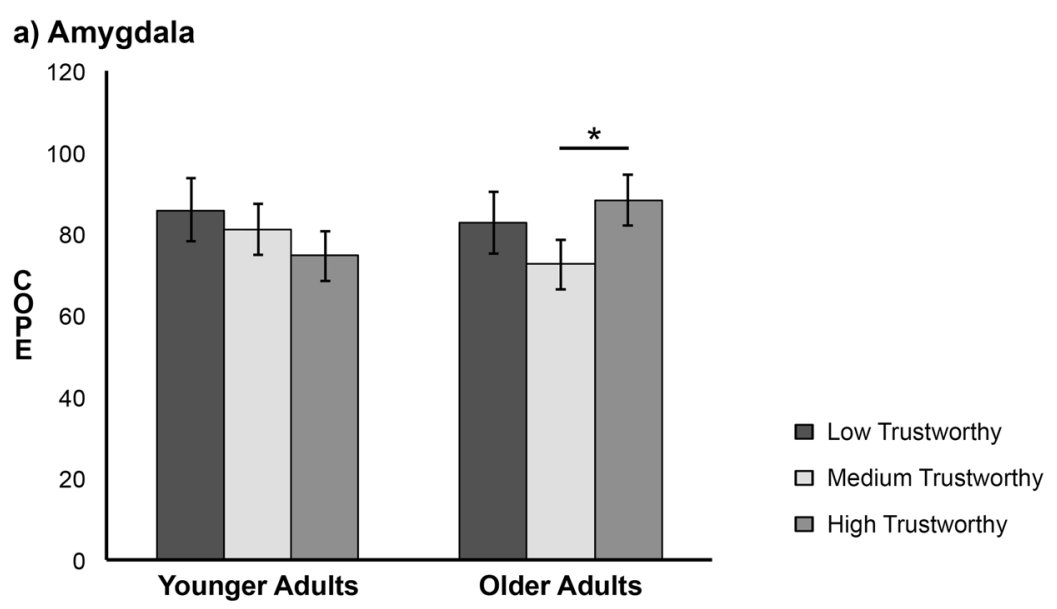

b) Caudate

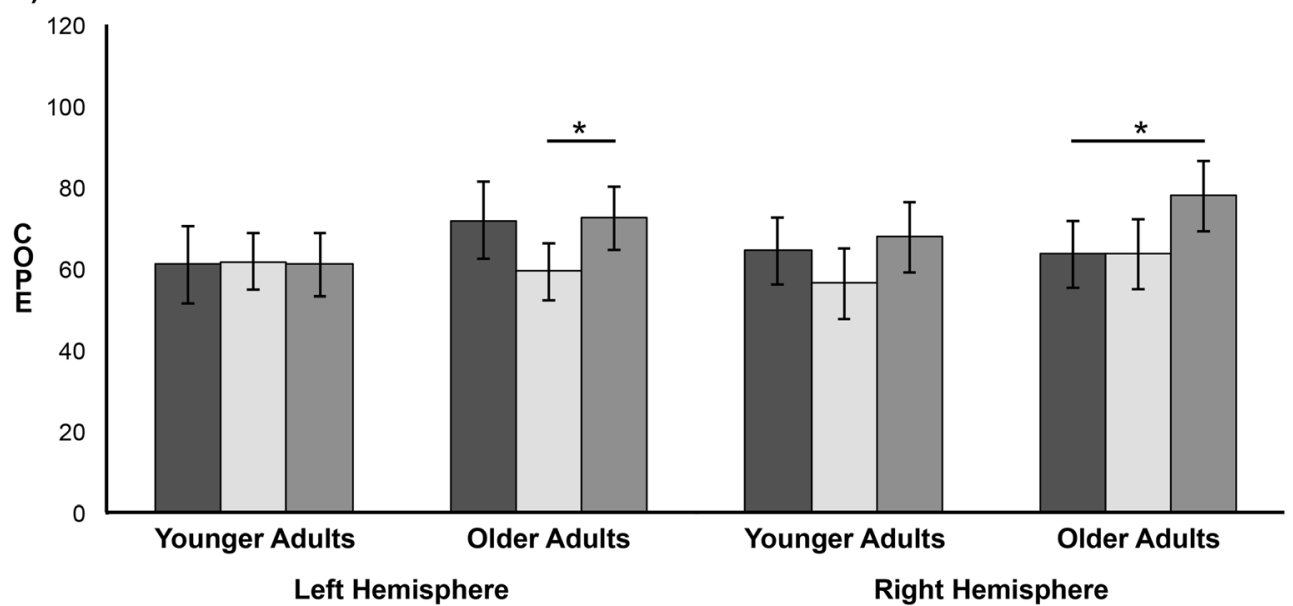

Fig. 1 Peak activation as a function of face trustworthiness for younger (YA) and older $(\mathrm{OA})$ participants in ROIs that showed interactions of face trustworthiness with participant age: a amygdala; $\mathrm{b}$ caudate. Error bars

represent standard errors. COPE is the contrast of parameter estimates (high or medium or low trustworthy faces minus baseline fixation) from which peak values were extracted at the subject-level using FSL featquery. $* p<.05$

\section{$d A C C$}

As predicted, there was a significant main effect for face trustworthiness, reflecting stronger dACC activation to high face trustworthiness than low face trustworthiness, $p=.007$, consistent with Hypothesis 4, with no difference between medium face trustworthiness and high or low face trustworthiness, respective $p s=.359$ and .109 . The participant age effect and the Age $\times$ Face Trustworthiness interaction were not significant. Hemisphere effects are not applicable to this ROI.

\section{Caudate}

The main effects of face trustworthiness and participant age and the Face Trustworthiness $\times$ Age interaction were all nonsignificant. There also was no significant main effect for hemisphere. However, a marginally significant Trustworthiness $\times$ Hemisphere effect was qualified by a significant Age $x$ Trustworthiness $\times$ Hemisphere interaction. Comparisons between face trustworthiness levels within each age and

hemisphere revealed no significant effects for YA, all $p$ s > .13. Consistent with Hypothesis 4, OA showed stronger activation to high than to medium face trustworthiness in left hemisphere, $p=.03$, and stronger activation to high than low face trustworthiness in right hemisphere, $p=.038$. OA showed no significant differences between low and medium face trustworthiness in either the left or right hemisphere, respective $p \mathrm{~s}=.104$ and .993 , between high and low face trustworthiness in the left hemisphere, $p \mathrm{~s}=.930$, and between high and medium face trustworthiness in the right hemisphere, $p=$ .064 (see Fig. 1b).

Insula

The main effects of face trustworthiness and participant age and the Face Trustworthiness $\times$ Age interaction were all nonsignificant. There also were no significant main effects or interaction effects on insula activation involving hemisphere. 


\section{$m O F C$}

The main effects of face trustworthiness and participant age, and the Face Trustworthiness $\times$ Age interaction were all nonsignificant, although there was a nonsignificant trend for face trustworthiness in the direction predicted by Hypothesis 4. A significant effect of hemisphere revealed stronger mOFC activation in the right than in the left, but there were no interaction effects involving hemisphere.

\section{NAcc}

The main effects of face trustworthiness and participant age and the Face Trustworthiness $\times$ Age interaction were all nonsignificant, although there was a nonsignificant trend for face trustworthiness in the direction predicted by Hypothesis 4. There were no significant effects on NAcc activation involving hemisphere.

\section{$v m P F C$}

The main effects of face trustworthiness and participant age and the Face Trustworthiness $\times$ Age interaction were all nonsignificant. There was a main effect for hemisphere, reflecting stronger vmPFC activation on the right than on the left, but none of the interactions with hemisphere were significant.

\section{Trustworthy ratings}

We correlated the pretest ratings made by young adults on MTurk with trustworthy ratings by YA and OA participants to confirm that there were no age differences in the strength of the manipulation. The correlations showed large effects that were equally strong for YA, $r(178)=.858, p<.001$, and OA, $r(178)=.856, p<.001$. As the meta-analysis had aggregated studies investigating face attractiveness and trustworthiness, we also correlated pretest ratings of attractiveness with trustworthy ratings by YA and OA to confirm the discriminant validity of our manipulation. Although the correlations were significant, the effects were small for both YA, $r(178)=.182$, $p=.015$, and OA, $r(178)=.204, p=.006$.

We performed a 3 (face trustworthiness) $\times 2$ (participant age) $\times 2$ (face age) mixed ANOVA on trustworthy ratings, with face age and face trustworthiness as within-groups variables. Rating data were missing for one YA. A strong effect of face trustworthiness, $F(2,90)=196.33, p<.001, \eta^{2}=.814$, revealed significant differences between high $(M=4.94, S E=.12)$, medium $(M=4.22, S E=.12)$, and low $(M=3.49, S E=.13)$ face trustworthiness, all $p \mathrm{~s}<.001$. This effect was moderated by a significant Face Trustworthiness $\times$ Face Age effect, $F(2,90)=$ $17.22, p<.001, \eta^{2}=.277$. The differences between high, medium, and low trustworthy faces were significant within both older and younger faces, all $p \mathrm{~s}<.001$. What accounted for the interaction was that low trustworthy younger faces $(M=3.64$, $S E=.12)$ were rated as more trustworthy than low trustworthy older faces $(M=3.33, S E=.16), p=.003$, while there was no significant difference between the rated trustworthiness of younger and older faces in the medium or high trustworthy groups, respective $p s=.447$ and .169 (see Fig. 2). The threeway interaction with participant age was not significant, $F(2$, $88)=2.08, p=.131, \eta^{2}=.044$, indicating that rater age did not moderate the differences in trustworthiness of younger and older faces across the three conditions. As predicted, there also was a significant main effect of participant age, with OA giving higher trustworthy ratings $(M=4.64, S E=.17)$ than did YA $(M$ $=3.79, S E=.17), F(1,45)=12.67, p=.001, \eta^{2}=.220$. The nonsignificant Participant Age $\times$ Face Trustworthiness and Participant Age $\times$ Face Age interaction effects indicated that the OA positivity effect was not moderated by face trustworthiness, $F(2,90)=.50, p=.608, \eta^{2}=.011$, or face age, $F(1,45)=$ $.24, p=.625, \eta^{2}=.005$.

\section{Discussion}

This is the first study to show effects of face trustworthiness on OA neural activation, with significant effects exclusive to OA in the amygdala and caudate, and an effect that was not moderated by age in the dACC. As predicted by Hypothesis 1, the effects of face trustworthiness on activation in these reward network regions are consistent with results for YA reported in previous meta-analyses (Mende-Siedlecki et al., 2013; Santos et al., 2016). However, unlike the Mende-Siedlecki et al. (2013) meta-analysis, we found no significant effects of face trustworthiness on activation in the insula, mOFC, NAcc, or vmPFC.

Hypothesis 2 predicted that YA, but not OA, would show stronger activation to low than to high trustworthy faces in the amygdala and right insula. The predicted effects for YA were not significant, which makes it impossible to interpret the null effects that were predicted and found for OA. We found partial support for Hypothesis 3, which predicted that both YA and OA would show a nonlinear response to face trustworthiness in the amygdala, with the YA effect stronger for low versus medium trustworthiness and the OA effect stronger for high versus medium trustworthiness. A significant face trustworthiness by participant age effect revealed that, although there was no significant nonlinear effect for YA, OA showed significantly stronger activation to high than to medium trustworthy faces, no significant difference between low and medium, and a significant nonlinear effect, as predicted. These OA results are consistent with meta-analytic evidence for nonlinear amygdala responses to face trustworthiness/attractiveness in YA across many studies (Mende-Siedlecki et al., 2013; Santos et al. 2016). The frequent nonlinear response in the amygdala may explain why Castle et al. (2012) failed to find an amygdala response to trustworthiness in either OA or YA, 


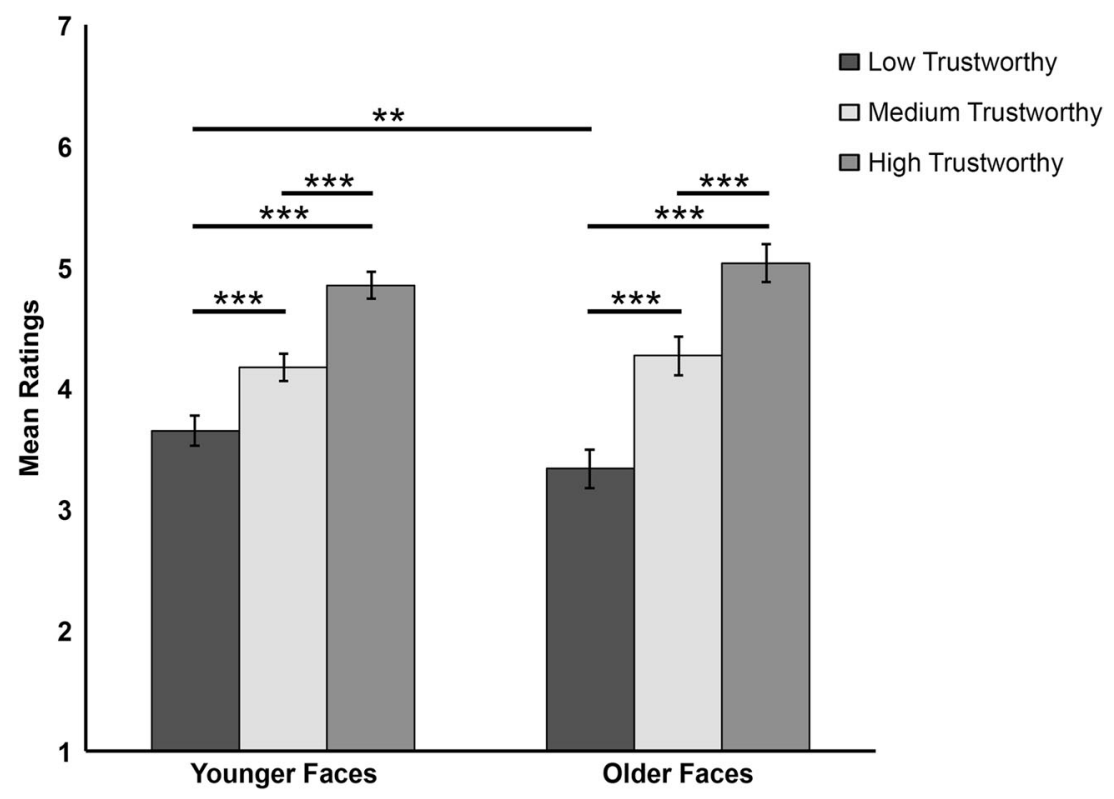

Fig. 2 Trustworthy ratings as a function of face trustworthiness category and face age. Error bars represent standard errors. $* * * p<.001 . * * p<.01$

since they only examined the linear effect of low versus high trustworthy faces.

The absence of significantly stronger $\mathrm{OA}$ amygdala activation to low than to medium trustworthy faces as well as to low than to high suggests that OA may be relatively insensitive to low trustworthiness. Although the nonlinear pattern shown by $\mathrm{OA}$ is consistent with that shown by YA in one study (Rule, Krendl, Ivcevic, \& Ambady, 2013), it differs from the YA pattern in other studies, which showed stronger effects for low versus medium than high versus medium trustworthiness in YA (Said, Baron, \& Todorov, 2008; Winston, Strange, O’Doherty, \& Dolan, 2002). Research reporting nonlinear amygdala activation to other emotionally evocative stimuli has also found that negative images elicit the strongest response in YA, while positive images elicit the strongest response in OA (Mather et al., 2004), consistent with the OA positivity effect. A definitive answer to the question of whether OA amygdala response is relatively insensitive to low face trustworthiness requires a study of OA and YA that shows significant amygdala effects for both age groups.

Hypothesis 4 predicted that both YA and OA would show stronger activation to high than to medium or low trustworthy faces in the dACC, caudate, left insula, mOFC, and vmPFC, and that these effects may be stronger for OA. Consistent with these predictions, a significant face trustworthiness effect in dACC revealed significantly stronger activation to high than low trustworthy faces, although neither differed from medium trustworthy faces. In addition, the absence of an interaction with participant age indicated that this effect did not differ significantly by age. On the other hand, a Trustworthiness $\times$ Participant Age $\times$ Hemisphere effect in the caudate revealed that $\mathrm{OA}$ showed stronger right caudate activation to high than to low trustworthy faces, and stronger left caudate activation to high than to medium, whereas there were no significant effects for YA. Although Hypothesis 4 predicted that this effect might be weaker for YA, we did not expect it to be absent. The effect for $\mathrm{OA}$ is consistent with research showing that smiling faces that signal reward elicit stronger OA and YA caudate activation than frowning faces that signal punishment (Drueke et al., 2015).

The absence of significant YA neural activation to face trustworthiness in the amygdala and caudate suggests that YA may not have shown effects in other ROIs. If so, this would contribute to the absence of significant main effects of trustworthiness across both age groups. A possible explanation for the failure of YA in our study to replicate the meta-analytic results that OA replicated in the amygdala and caudate is that YA are less sensitive to variations in trustworthiness in older faces. The metaanalyses documenting significant YA effects were based on studies that used only young adult faces, whereas half of the faces in our study were older faces. If this is the explanation, then it is important to note that the greater dACC activation to high than to low trustworthy faces did not vary with participant age. Thus, it seems that if YA do show lesser sensitivity than OA to trustworthiness variations in older faces, this may be restricted to subcortical activation.

As noted above, we deliberately included both older and younger faces to eliminate the possibility that stronger effects for one age group than the other might reflect the own-age bias that has been documented in behavioral research on face perception, with an own-age advantage in the case of identity, age, and emotion recognition (Anastasi \& Rhodes, 2005; Folster et al., 2015; Fulton \& Bartlett, 1991; Malatesta et al., 1987; Perfect \& Harris, 2003; Riediger et al., 2011; Voelkle et al., 2012; Wright \& Stroud, 2002), and an own-age disadvantage in the case of deception detection (Slessor et al., 2014). Notably, an own-age advantage also has been shown 
in greater neural activation to own-age than to other-age facial expressions in several reward regions (Ebner et al., 2013). It is possible that the "other-age disadvantage" was stronger for YA than for OA in the case of neural responses to variations in trustworthiness, thereby eliminating the significant YA effects that were obtained in past research and for OA in the present study. Consistent with this possibility, a study by Bos, Hermans, Ramsey, \& van Honk (2012) that included both older and younger faces found no significant amygdala response to low face trustworthiness among YA participants. Also, although we have argued that Castle et al. (2012) may have found no significant effects of face trustworthiness on amygdala activation because their design did not permit a test of nonlinear effects, the inclusion of older faces also may have contributed to this result in the case of YA. On the other hand, Castle et al. (2012) did find significant insula responses to face trustworthiness in YA despite including older faces. Because it is unclear whether face age was balanced across the different levels of trustworthiness in that study, it is possible that YA showed an insula response to those lowest in trustworthiness because most were younger faces. Unfortunately, the block design of our study, which included in each block both older and younger faces of the same trustworthiness, prevents us from examining neural activation to older versus younger faces considered separately. Future research should address this question. The block design also prevented us from taking a parametric approach to examining the relationship between face trustworthiness and neural activation. However, we chose this approach because a block design is generally more powerful for detecting effects than event-related designs.

The fact that we included only female faces might also conceivably account for the finding that OA showed significant neural sensitivity to variations in trustworthiness that YA did not display. We examined 25 studies to assess the gender composition of faces in past research: Castle et al. (2012) plus 24 unique studies in the reviews by Santos et al. (2016) and Mende-Siedlecki et al. (2013). Of these, 12 used faces of both sexes, three used only male faces, five used computergenerated faces, and five did not specify face sex. Eight of the mixed-sex studies systematically varied trustworthiness within faces of each sex but did not examine the effects within sex. Thus, it is possible that effects of trustworthiness documented in these studies were carried by the male faces. However, we know of no theory or research suggesting that face sex would differentially moderate OA and YA neural responses to trustworthiness. Four of the mixed-sex studies did not control the distribution of male and female faces across trustworthiness. As noted in our discussion of the Castle et al. study, this may confound face trustworthiness with face sex. The same problem arises for the five studies that used computer-generated faces for which low trustworthy faces resemble males and high trustworthy faces resemble females (Todorov, 2017). Thus, it is possible that effects of face trustworthiness and perceived face sex were confounded in these studies. It would be useful for future research to investigate these possibilities.

Another possible explanation for our failure to replicate the meta-analytic effects of face trustworthiness in several ROIs is that our study was underpowered. As noted in the Method section, the one study that included both younger and older adults (Castle et al., 2012) and 20 of the 24 studies examining trustworthiness in the literature reviews (Mende-Siedlecki et al., 2013; Santos et al., 2016) did not report statistics from which a power analysis could be performed. The results for the remaining four studies revealed an estimated sample size per group of 27 in a two-group repeated-measures high/low trustworthiness design for $80 \%$ power at a .05 alpha level. Trustworthiness was also a repeated measure in our design, and our sample size was 48 per face trustworthiness group, summing across participant age. Given the small set of studies from which power was calculated, it is noteworthy that our sample size also was greater than the average sample size across the 24 unique studies of trustworthiness in the Mende-Siedlecki et al. (2013) and the Santos et al. (2016) reviews $(M=20.08)$ and the sample size in the Castle et al. (2012) study ( $N=21 \mathrm{YA} ; N=23 \mathrm{OA})$. It was also greater than the Drueke et al. (2015) study that found differential YA and OA activation to smiling versus frowning faces in NAcc, among other regions, with $N=16$ in each age group. Thus, based on the available information, it does not seem that our study was underpowered. Most important, when comparing our results to Castle et al. (2012), power differences seem unable to explain the pattern of results. They found effects in insula for YA, but not OA, and no effects in the amygdala. We found effects in the amygdala for OA but not for YA, and no effects in insula. Nevertheless, it may be that a larger sample size would increase power sufficiently to yield significant positive effects of face trustworthiness on $\mathrm{mOFC}$ and NAcc activation, as well as a significant negative effect on YA amygdala activation, all of which were marginally significant in our study.

As predicted by Hypothesis 5, OA ratings of face trustworthiness were more positive than those of YA, consistent with other evidence for an OA positivity effect in trait ratings of faces (Castle et al., 2012; Zebrowitz et al., 2017; Zebrowitz, et al., 2013). One might suggest that these age differences derive from the fact that we determined the trustworthiness of the faces based on ratings by an independent group of YA. However, the correlations between the pretest ratings and YA and OA participants' own ratings were equally high. Moreover, one might expect that selecting faces based on independent ratings from YA would yield stronger neural effects of manipulated face trustworthiness for YA than OA. Yet we found effects for OA, and not for YA.

One can ask what are the implications of our findings for the question of whether age differences in neural activation to 
variations in face trustworthiness might render OA more trusting and possibly more vulnerable to fraud? Although $\mathrm{OA}$ in our study were more trusting in the sense that they perceived all faces as more trustworthy than YA did, their ratings nevertheless showed sensitivity to differences between high, medium, and low trustworthy faces equal to that shown by YA. On the other hand, OA showed neural responses in the reward circuit that distinguished high trustworthy faces from medium and low trustworthy faces, albeit not medium from low. To the extent that neural activation in these reward regions subserves approach/avoidance responses, OA should show the greatest approach to people who look highly trustworthy, and equal approach to people who look medium and low in trustworthiness. This could mean either that OA are overly trusting of people who look low in trustworthiness or overly wary of those who look medium in trustworthiness. To address these questions requires research that employs behavioral measures that are more sensitive to the approachavoidance responses informed by the neural reward network than are trustworthy ratings. Useful in this regard would be paradigms that assess mouse trajectories when approaching faces (Martens et al., 2012), joystick responses that push faces away from perceivers or pull them closer (Enter, Colzato, \& Roelofs, 2012), or responses in economic games. Research using the latter paradigm has revealed that YA and OA show an equal tendency to invest more money with high than with low-trustworthy-looking people (Bailey et al., 2016), and that YA and OA do not differ in overall trusting behavior as indexed by monetary investments (Bailey et al., 2015). However, the critical comparison suggested by our research is age differences in trusting behavior toward those who look low versus medium in trustworthiness, and this remains to be determined.

Of course, favoring more trustworthy-looking people can protect against fraud only to the extent that such people are in fact more trustworthy. Several studies indicate that YA judgments of trustworthiness do not accurately differentiate high from low trustworthy people, as indexed by whether they have a criminal history or had cheated on a test (Rule et al., 2013). Most relevant to the present research is that YA amygdala activation was greater for faces judged as untrustworthy but not for faces of people who had actually cheated. However, cheating on an exam is only one facet of trustworthiness, and it has less salient interpersonal ramifications than some other behaviors, like aggression and exploitation. In fact, research has demonstrated equal YA and OA accuracy in judging aggressiveness (Boshyan, Zebrowitz, Franklin, McCormick, \& Carre, 2014), as well as accuracy in judging violent criminal behavior in a study of YA (Boshyan, 2016). Other research showed that facial shape was a valid predictor of untrustworthiness, as indexed by the extent to which someone exploited others in an economic game. It also showed that facial shape influenced the extent to which YA trusted partners when playing the same game (Stirrat \& Perrett, 2010), suggesting that people may be able to discern the risk of exploitation. Although OA were not included in that study, another study showed that both OA and YA used the same face-shape cue that was diagnostic of exploitation to accurately identify aggressiveness (Boshyan et al., 2014). Thus, the current finding that neural responses to high trustworthy faces are intact in OA provides some reason to believe that OA neural activation would respond to variations in faces that signal actual trustworthiness.

\section{Summary and conclusions}

This is the first study to compare OA and YA neural activation to faces varying in trustworthiness across all regions in the reward circuit that were previously shown to respond in YA. Our results reveal an OA neural response to face trustworthiness. Specifically, we found a significant nonlinear response to face trustworthiness in the amygdala and a positive linear response in the caudate that were exclusive to $\mathrm{OA}$, as well as a positive linear response in the dACC that was not moderated by age. The significant amygdala effect for OA contrasts with results reported by Castle et al. (2012) and may reflect our use of an experimental design that is sensitive to nonlinear as well as linear effects. Our finding that YA did not show significant amygdala and caudate sensitivity to face trustworthiness is surprising given that these effects have been well-documented in previous research. The possibility that this was due to our inclusion of equal numbers of older and younger faces should be pursued in future research. Also important is research designed to determine whether the neural response to faces is sensitive to actual differences in trustworthiness and whether this is related to meaningful behavioral responses across age. Such research will shed further light on the neural mechanisms for any age differences in vulnerability to fraud.

Acknowledgements This research was funded by National Institutes of Health Grant AGO38375 to the first author. The authors would like to thank Luke Hanlin and Eri Ichijo for their help with fMRI scanning and behavioral data collection.

\section{References}

Anastasi, J. S., \& Rhodes, M. G. (2005). An own-age bias in face recognition for children and older adults. Psychonomic Bulletin \& Review, 12, 1043-1047.

Bailey, P. E., Slessor, G., Rieger, M., Rendell, P. G., Moustafa, A. A., \& Ruffman, T. (2015).Trust and trustworthiness in young and older adults. Psychology and Aging, 30, 977-986.

Bailey, P. E., Szczap, P., McLennan, S. N., Slessor, G., Ruffman, T., \& Rendell, P. G. (2016). Age-related similarities and differences in 
first impressions of trustworthiness. Cognition and Emotion, 30, 1017-1026.

Benton, A., Van Allen, M., Hamsher, K., \& Levin, H. (1983). Test of facial recognition manual. Iowa City, IA: Benton Laboratory of Neuropsychology.

Bos, P. A., Hermans, E. J., Ramsey, N. F., \& van Honk, J. (2012). The neural mechanisms by which testosterone acts on interpersonal trust. NeuroImage, 61, 730-737.

Boshyan, J. (2016). In your face: Examining mechanisms of accurately perceiving threat from faces (Doctoral dissertation). Retrieved from ProQuest Dissertations and Theses database (UMI No. 10157892).

Boshyan, J., Zebrowitz, L. A., Franklin, R. G. J., McCormick, C. M., \& Carre, J. M. (2014). Age similarities in recognizing threat from faces and diagnostic cues. Journal of Gerontology: Psychological Sciences and Social Sciences, 69(5), 710-718.

Buckner, R. L., Synder, A. Z., Sanders, A. L., Raichle, M. E., \& Morris, J. C. (2000). Functional brain imaging of young, nondemented, and demented older adults. Journal of Cognitive Neuroscience, 12(Suppl. 2), 24-34.

Carrns, A. (2017). Why older people are vulnerable to fraud, and how to protect them. The New York Times. Retrieved from https://www. nytimes.com/2017/02/17/your-money/why-older-people-arevulnerable-to-fraud-and-how-to-protect-them.html

Castle, E., Eisenberger, N. I., Seeman, T. E., Moons, W. G., Boggero, I. A., Grinblatt, M. S., \& Taylor, S. E. (2012). Neural and behavioral bases of age differences in perceptions of trust. Proceedings of the National Academy of Sciences of the United States of America. 4109, 2088-20852. https://doi.org/10.1073/pnas.1218518109

Czerwon, B., Lüttke, S., \& Werheid, K. (2011). Age differences in valence judgments of emotional faces: The influence of personality traits and current mood. Experimental Aging Research, 37, 503-515.

D’Esposito, M., Zarahn, E., Aguirre, G. K., \& Rypma, B. (1999). The effect of normal aging on the coupling of neural activity to the bold hemodynamic response. NeuroImage, 10, 6-14.

Delgado, M. R. (2007). Reward-related responses in the human striatum. In B. W. Balleine, K. Doya, J. Odoherty, \& M. Sakagami (Eds.), Reward and decision making in corticobasal ganglia networks (Vol. 1104, pp. 70-88). New York, NY: New York Academy of Sciences.

Desikan, R. S., Ségonne, F., Fischl, B., Quinn, B. T., Dickerson, B. C., Blacker, D., . ... Killiany, R. J. (2006). An automated labelling system for subdividing the human cerebral cortex on MRI scans into gyral based regions of interest. Neuroimage, 31(3), 968-980.

Drueke, B., Weichert, L., Forkmann, T., Mainz, V., Gauiggel, S., \& Boecker, M. (2015). Neural correlates of positive and negative performance feedback in younger and older adults. Behavioral and Brain Functions, 11, 17. https://doi.org/10.1186/s12993-015-0062-Z

Ebner, N. C., Johnson, M. R., Rieckmann, A., Durbin, K., Johnson, M. K., \& Fischer, H. (2013). Processing own-age vs. other-age faces: Neuro-behavioral correlates and effects of emotion. NeuroImage, 78, 363-371. https://doi.org/10.1016/j.neuroimage.2013.04.029

Elliott, R., Newman, J. L., Longe, O. A., \& Deakin, J. F. W. (2003). Differential response patterns in the striatum and orbitofrontal cortex to financial reward in humans: A parametric functional magnetic resonance imaging study. Journal of Neuroscience, 23. 303-307.

Enter, D., Colzato, L. S., \& Roelofs, K. (2012). Dopamine transporter polymorphisms affect social approach-avoidance tendencies. Genes, Brain and Behavior, 11, 671-676.

Folstein, M. F., Folstein, S. E., \& McHugh, P. R. (1975). Mini-Mental State: A practical method for grading the cognitive state of patients for the clinician. Journal of Psychiatric Research, 12, 189-198.

Folster, M., Hess, U., Huhnel, I., \& Werheid, K. (2015). Age-related response bias in the decoding of sad facial expressions. Behavioral Science, 5, 443-460.
Fulton, A., \& Bartlett, J. C. (1991). Young and old faces in young and old heads-The factor of age in face recognition. Psychology and Aging, 6, 623-630.

Hadjikhani N, Zurcher N, Lassalle A, Hippolyte L, Ward N, ÅsbergJohnels J. (2017). The effect of constraining eye-contact during emotional face perception - an fMRI study. Social Cognitive and Affective Neuroscience, 12, 1197-1207.

Hayes, D.J., Duncan, N.W., Xu, J., \& Northoff, G. (2014). A comparison of neural responses to appetitive and aversive stimuli in humans and other animals. Neuroscience and Biobehavioral Reviews, 45, 350368.

Hehman, E., Stolier, R. M. \& Freeman, J. B. (2015). Advanced mousetracking analytic techniques for enhancing psychological science. Group Processes and Intergroup Relations, 18, Special Issue: SI, 384-401.

Hosokawa, T., Kato, K., Inoue, M., \& Mikami, A. (2007) Neurons in the macaque orbitofrontal cortex code relative preference of both rewarding and aversive outcomes. Neuroscience Research, 57, 434445.

Isaacowitz, D. M., \& Blanchard-Fields, F. (2012). Linking process and outcome in the study of emotion and aging. Perspectives on Psychological Science, 7, 3-17.

Kensinger, E. A. (2008). Age differences in memory for arousing and nonarousing emotional words. Journal of Gerontology: Psychological Sciences, 63B, 13-18.

Liang, X., Zebrowitz, L. A., \& Zhang, Y. (2010). Neural activation in the 'reward circuit' shows a nonlinear response to facial attractiveness. Social Neuroscience, 5, 320-334

Liu, X., Hairston, J., Schrier, M., \& Fan, J. (2011). Common and distinct networks underlying reward valence and processing stages: A metaanalysis of functional neuroimaging studies. Neuroscience and Biobehavioral Reviews, 35, 1219-1236.

Malatesta, C. Z., Izard, C. E., Culver, C., \& Nicolich, M. (1987). Emotion communication skills in young, middle-aged, and older women. Psychology and Aging, 2, 193-203.

Martens, M. A., Hasinski, A. E., Andridge, R. R., \& Cunningham, W. A. (2012). Continuous cognitive dynamics of the evaluation of trustworthiness in Williams syndrome. Frontiers in Psychology, 3(160). https://doi.org/10.3389/fpsyg.2012.00160

Mather, M., Canli, T., English, T.,Whitfield, S., Wais, P., Ochsner, K., Gabrieli, J. D. E., \& Carstensen, L. L. (2004). Amygdala response to emotionally valenced stimuli in older and younger adults. Psychological Science, 15, 259-263.

Mende-Siedlecki, P., Said, C. P., \& Todorov, A. (2013). The social evaluation of faces: A meta-analysis of functional neuroimaging studies. Social Cognitive Affective Neuroscience, 8, 285-299.

Murphy, N. A. \& Isaacowitz, D. M. (2008). Preferences for emotional information in older and younger adults: A meta-analysis of memory and attention tasks. Psychology and Aging. 23, 263-86.

Namburi, P. , Al-Hasani, R.,Calhoon, G. G., Bruchas, M.R., \& Tye, K. M. (2016). Architectural representation of valence in the limbic system. Neuropsychopharmacology, 41, 1697-1715.

Nishijo, H., Ono, T., \& Nishino, H. (1988). Single neuron responses in amygdala of alert monkey during complex sensory stimulation with affective significance. The Journal of Neuroscience : The Official Journal of the Society for Neuroscience, 8, 3570-3583.

Oliveira, D., Rocha, H., Yang, H., Ellis, D., Dommaraju, S., Muradoglu, M.,. .. Ebner, N. (2017). Dissecting spear phishing emails for older vs young adults: On the interplay of weapons of influence and life domains in predicting susceptibility to phishing. $\mathrm{CHI}$ '17: $\mathrm{CHI}$ Conference Proceedings on Human Factors in Computing Systems, 6412-6424. https://doi.org/10.1145/10.1145/3025453. 3025831

Perfect, T. J., \& Harris, L. J. (2003). Adult age differences in unconscious transference: Source confusion or identity blending? Memory \& Cognition, 31, 570-580. 
Piper, B. J., Li, V., Eiwaz, M. A., Kobel, Y. V., Benice, T. S., Chu, A. M.,. .. Mueller, S. T. (2012). Executive function on the psychology experiment building language tests. Behavior Research Methods, 44, $110-123$.

Reed, A. E., \& Carstensen, L. L. (2012). The theory behind the agerelated positivity effect. Frontiers in Psychology, 3(339). https:// doi.org/10.3389/psyg.2012.00339

Reed, A. E., Chan, L., \& Mikels, J. A. (2014). Meta-analysis of the agerelated positivity effect: Age differences in preferences for positive over negative information. Psychology and Aging, 29, 1-15.

Riediger, M., Voelke, M. C., Ebner, N. C., \& Lindenberger, U. (2011). Beyond 'happy, angry, or sad': Age-of-poser and age-of-rater on multi-dimensional emotion perception. Cognition and Emotion, $25,968-982$.

Ross, M., Grossman, I., \& Schryer, E. (2014). Contrary to psychological and public opinion, there is no compelling evidence that older adults are disproportionately victimized by consumer fraud. Perspectives on Psychological Science, 9, 427-442.

Ruffman, T., Murray, J., Halberstadt, J., \& Vader, T. (2012). Age-related differences in deception. Psychology and Aging, 27, 543-549. https://doi.org/10.1037/a0023380

Ruffman, T., Sullivan, S., \& Edge, N. (2006). Differences in the way older and younger adults rate threat in faces but not situations. Journal of Gerontology: Psychological Sciences, 61, 187-194.

Rule, N. O., Krendl, A. C., Ivcevic, Z., \& Ambady, N. (2013). Accuracy and consensus in judgments of trustworthiness from faces: Behavioral and neural correlates. Journal of Personality and Social Psychology, 104, 409-426. https://doi.org/10.1037/a0031050

Said, C. P., Baron, S. G., \& Todorov, A. (2008). Nonlinear amygdala response to face trustworthiness: Contributions of high and low spatial frequency information. Journal of Cognitive Neuroscience, 21, 519-528.

Salthouse, T. A. (1993). Speed and knowledge as determinants of adult age differences in verbal tasks. Journal of Gerontology, 48, 29-36.

Santos, S., Albeida, I., Oliveiros, B., \& Castelo-Branco, M. (2016). The role of the amygdala in facial trustworthiness processing: A systematic review and meta-analyses of fMRI studies. PLOS One 11(11), e0167276. https://doi.org/10.1371/journal.pone.0167276

Sescousse, G., Caldu, X., Segura, B., \& Dreher, J.-C., (2013). Preocessing of primary and secondary rewards: A quantitative meta-analysis and review of hyuman functional newuroimaging studies. Neuroscience and Biobehavioral Reviews, 37, 681-696.

Shipley, W. C. (1946). Institute of Living Scale. Los Angeles, CA: Western Psychological Services.
Slessor, G., Phillips, L. H., Ruffman, T., Bailey, P. E., \& Insch, P. (2014). Exploring own-age biases in deception detectioin. Emotion, 28, 493-506.

Smith, D. P., Hillman, C. H., \& Duley, A. R. (2005). Influences of age on emotional reactivity during picture processing. Journal of Gerontology Psychologicaln, 60B, 49-56.

Smith, S. (2002). Featquery - FEAT results interrogation user guide. Oxford, UK: University of Oxford Press.

Stanley, J. T., \& Blanchard-Fields, F. (2008). Challenges older adults face in detecting deceit: The role of emotion recognition. Psychology and Aging, 23(1), 24-32.

Stirrat, M., \& Perrett, D. I. (2010). Valid facial cues to cooperation and trust. Psychological Science, 21, 349-354.

Todorov, A. (2017). Face value: The irresistible influence of first impressions. Princeton, NJ: Princeton University Press.

Violations Against Elderly or Disabled Persons, Wisc. Code $\S 100.264$ (2012 through Act 45). Retrieved from http://docs.legis.wisconsin. gov/statutes/statutes/100/264

Voelkle, M. C., Ebner, N. C., Lindenberger, U., \& Riediger, M. (2012). Let me guess how old you are: Effects of age, gender, and facial expression on perceptions of age. Psychology and Aging, 27, 265277. https://doi.org/10.1037/a0025065

Watson, D., Clark, L. A., \& Tellegen, A. (1988). Development and validation of brief measures of positive and negative affect: The PANAS scales. Journal of Personality and Social Psychology, 54, 1063-1070.

Winston, J. S., O’Doherty, J., Kilner, J. M., Perrett, D. I., \& Dolan, R. J. (2007). Brain systems for assessing facial attractiveness. Neuropsychologia, 45, 195-206.

Winston, J. S., Strange, B. A., O’Doherty, J., \& Dolan, R. J. ( 2002). Automatic and intentional brain responses during evaluation of trustworthiness of faces. Nature Neuroscience, 5, 277-283. https:// doi.org/10.1038/nn816

Wright, D. B., \& Stroud, J. N. (2002). Age differences in lineup identification accuracy: People are better with their own age. Law and Human Behavior, 26, 641-654.

Zebrowitz, L. A., Boshyan, J., Ward, N., Gutchess, A. \& Hadjikhani, N. (2017). The older adult positivity effect in evaluations of trustworthiness: Emotion regulation or cognitive capacity? PLOS ONE. https://doi.org/10.1371/journal.pone.0169823

Zebrowitz, L. A., Franklin, R. G., Hillman, S., \& Boc, H. (2013). Older and younger adults' first impressions from faces: Similar in agreement but different in positivity. Psychology and Aging. 28, 202-212. https://doi.org/10.1037/a0030927 